\title{
Virtual Coach: un ambiente de simulación situacional para la enseñanza de la gerencia de la construcción
}

\section{Virtual Coach: a situational simulation environment for construction management education}

\author{
Eddy M. Rojas*, Amlan Mukherjee**
}

\author{
* U niversity of Washington, Seattle, ESTAD OS UN ID OS \\ ** M ichigan Technological University, Michigan, ESTAD OS U N ID OS \\ er@eu.washington.edu
}

Fecha de recepción: 29/ 12/2006

PAG. $25-32$

Resumen

\begin{abstract}
El modelo educativo tradicional utilizado en el área de gerencia de la construcción, basado en problemas precisos y claros y definiciones formales, no está cumpliendo con su misión de educar a los encargados de la toma de decisiones del mañana. Esta realización ha llevado a varios investigadores a explorar alternativas donde los problemas se resuelven dentro de su contexto natural y los conceptos promueven el aprendizaje dentro del nexo de la actividad. Varios esfuerzos se han realizado para desarrollar estos ambientes creando una variedad de simulaciones situacionales de propósito especial. Sin embargo, las simulaciones situacionales de propósito especial exhiben limitaciones inherentes relacionadas con su alcance, flexibilidad, y promoción del uso de colaboraciones. Estas limitaciones no se pueden resolver en el marco de ambientes con propósito especial. Un ambiente de uso general es requerido para superar estos problemas y tomar ventaja completa del modelo de aprendizaje situacional. Este artículo presenta aspectos teóricos relacionados con ambientes de simulación situacional como base conceptual para la introducción de un sistema de cómputo piloto llamado "Virtual Coach" (Entrenador Virtual). La implementación y la evaluación preliminar de este programa de cómputo son también discutidas en este artículo.
\end{abstract}

Palabras Clave: Simulación, modelos, gerencia de la construcción, enseñanza de la ingeniería, enseñanza asistida por computadora

Abstract

The traditional construction education model based on precise, well-defined problems and formal definitions is not satisfactorily fulfilling its mission of educating the decision-makers of tomorrow. This realization has moved several researchers to explore alternatives where problem solving is carried out in conjunction with the environment and concepts are embedded in the context promoting learning within the nexus of the activity. Several efforts have been undertaken to develop these environments resulting in a variety of special-purpose situational simulations. However, special-purpose situational simulations exhibit inherent limitations related to their application breadth, flexibility, and promotion of collaborations. These limitations cannot be resolved within the framework of special-purpose learning environments. A general-purpose environment is required to overcome these shortcomings and take full advantage of the situational learning paradigm. This paper presents theoretical aspects related to situational simulation environments as the conceptual framework to introduce a pilot application called Virtual Coach. The implementation and preliminary evaluation of this software system are also discussed in this paper.

Keywords: Simulation, models, construction management, engineering education, computer aided instruction

\section{Introducción}

La introducción de aspectos auténticos de la práctica profesional en la enseñanza de la gerencia de la construcción siempre ha sido difícil. McCabe et al., (2000) sostienen que el currículo actual del ingeniero civil enseña solamente las teorías de la gerencia de la construcción y que los estudiantes pueden encontrar dificultades al aplicar estos principios teóricos cuando están expuestos a las situaciones reales del mundo cotidiano. Sawhney et al., (2001) sostienen que los planes de estudios de ingeniería civil generalmente no permiten la inclusión de aplicaciones de importancia para la construcción, ni la participación en ejercicios clínicos o de experiencias prácticas. AbouRizk y Sawhney (1994), reconocen que los métodos de enseñanza tradicionales no son completamente capaces de proveer a los estudiantes con todas las habilidades necesarias para solucionar los problemas del mundo real encontrados en la construcción. Si la enseñanza de la gerencia de la construcción no provee a los estudiantes con las habilidades para solucionar problemas "del mundo real" 
o para aplicar conceptos teóricos a la práctica, entonces uno podría cuestionar la eficacia de los problemas estudiados o de las metodologías aplicadas.

La educación tradicional de la gerencia de la construcción sigue el modelo dualístico cartesiano de mente-materia (Barab et al., 2001) en donde se separa al estudiante del contexto del aprendizaje. Bajo este modelo, los conceptos se presentan como entidades fijas, bienestructuradas, e independientes. Las actividades se divorcian de su contexto auténtico dando por resultado la fragmentación y la especialización de cursos y de experiencias educativas. Esta fragmentación del conocimiento se ha identificado en el área de la construcción (Chinowsly y Vanegas, 1996; Fruchter, 1997) y es parcialmente responsable por la polarización del estudiante y del contexto en que se aprende. El conocimiento descontextualizado es intrínsicamente frágil tal como lo demuestran estudiantes que son capaces de recordar información para un examen, pero son incapaces de aplicar los mismos conceptos en la práctica profesional incluso cuando la situación claramente amerita tal acción (Brown et al., 1989). O tro ejemplo de esta fragilidad se puede observar en la universidad cuando los estudiantes ni retienen ni pueden utilizar los conocimientos que supuestamente adquirieron en cursos anteriores (Bert y Backer, 1996). Estos problemas, por supuesto, no son exclusivos a la educación en el área de la construcción, pero son compartidos por la mayoría de los modelos de educación superior.

La separación del estudiante del contexto del aprendizaje puede hacer que el conocimiento en sí mismo se vuelva inerte e inaplicable debido a la eliminación de la complejidad natural del contenido, la simplificación excesiva de relaciones, y la ausencia de la investigación auténtica del problema; creando los efectos secundarios de extinguir la creatividad y disminuir el entusiasmo (Barab et al., 2001). Ejemplos ilustrativos y visitas de campo al sitio de la obra han sido utilizados por la facultad de los programas de construcción como medios de generar conocimientos prácticos y robustos con un éxito parcial. Sin embargo, los ejemplos ilustrativos pueden dar la impresión de que existen soluciones fáciles y respuestas universalmente correctas debido a las simplificaciones necesarias (Pennell et al., 1997) y visitas de campo al sitio de la obra, especialmente aquellas que involucran grupos de un tamaño considerable, pueden no ser bienvenidas, incluir riesgos, y ser imprácticas desde el punto de vista logístico (Echeverri, 1996).

El modelo educativo tradicional utilizado en el área de gerencia de la construcción, basado en problemas precisos y claros y definiciones formales, no está cumpliendo con su misión de educar a los encargados de la toma de decisiones del mañana. Esta realización ha llevado a varios investigadores a explorar alternativas donde los problemas se resuelven dentro de su contexto natural y los conceptos promueven el aprendizaje dentro del nexo de la actividad. Estos esfuerzos han conducido a la generación de juegos y simulaciones tales como Superbid (AbouRizk, 1993), STRATEGY (McCabe et al., 2000), y VIRCON (Jaafari et al., 2001). Estos sistemas constituyen una evolución natural de esfuerzos anteriores tales como CONSTRU CTO (Halpin, 1970) y ARO USAL (N dekugri y Lansley, 1992). Estas simulaciones situacionales de propósito especial representan el primer paso hacia la puesta en práctica de un modelo participativo y contextualmente comprensivo en el área de la educación de la construcción. Las simulaciones de propósito especial, sin embargo, presentan varias limitaciones incluyendo su alcance limitado debido a su carácter restrictivo, su reducida flexibilidad debido a la carencia de programabilidad, y su inhabilidad de promover la creación de nuevas simulaciones y colaboraciones. Este artículo introduce un ambiente virtual de simulaciones situacionales de uso general aplicable al área de la educación en construcción con el objeto de superar estas limitaciones.

\section{Relevancia: la necesidad por un ambiente de propósito general}

El aprendizaje situacional puede definirse como el método pedagógico constructivista donde los estudiantes construyen su entendimiento al interactuar con ambientes enriquecidos que incentivan la explicación y el descubrimiento y en donde los profesores cambian su papel de trasmitir las "respuestas correctas" a los estudiantes a dirigir sus acciones en las actividades que incorporan representaciones personalmente significativas y prácticamente funcionales (Barab et al., 2001). En ambientes de aprendizaje situacionales los conocimientos se combinan intrínsecamente con su contexto, incentivando la comprensión conceptual a través de la evaluación de paradojas aparentes y relaciones que no son evidentes. Este modelo es claramente antagonista a la actividad típica de la universidad y puede incluso representar un estigma para aquellos acostumbrados a los modelos didácticos basados en clases magistrales. Sin embargo, el uso de métodos de aprendizaje activos ha sido reconocido como una estrategia fundamental 
para la reestructuración de la educación de la ingeniería (NSF 1995) dado que los estudiantes aprenden más eficazmente y retienen mejor sus conocimientos cuando pueden participar activamente en el proceso de aprendizaje (Chi et al., 1989).

Las simulaciones situacionales pueden ser de propósito específico o de uso general. Sin embargo, las simulaciones situacionales de propósito específico exhiben limitaciones inherentes relacionadas con su alcance, flexibilidad, y promoción del uso de colaboraciones. Estas limitaciones no se pueden resolver en el marco de estos ambientes de aprendizaje de propósito específico. Un ambiente de uso general es necesario para superar estos problemas y para tomar ventaja absoluta del modelo de aprendizaje situacional. En términos de su alcance, las simulaciones situacionales de propósito específico se enfocan en un área específica. Esta área está circunscrita generalmente por los proyectos y los casos seleccionados por el programador. Es decir, los sistemas basados en este modelo no se pueden ampliar fácilmente sin re-escribir sus programas de cómputo. Las situaciones experimentadas por los estudiantes también se limitan a los casos que se han programado dentro de la herramienta. Las simulaciones situacionales de uso general, por otra parte, son aplicables en un dominio muy amplio y se pueden utilizar para modelar casi cualquier clase de situación. No hay necesidad de modificar sus programas de cómputo para expandir su aplicabilidad.

Con respecto a flexibilidad, las simulaciones situacionales de propósito especial son limitadas por la construcción o el formalismo matemático usado debido a su carencia de programabilidad. Una simulación situacional de uso general, en contraste, permite que los modeladores definan sus propias formulaciones sin limitaciones con la excepción de las variables apoyadas por el modelo general.

Las simulaciones situacionales de uso general pueden promover colaboraciones de maneras sinérgicas que no son posibles en las simulaciones situacionales de propósito específico. Los modelos de uso general pueden facilitar la colaboración entre instituciones de educación superior y entre la comunidad académica y la industria para tomar ventaja de todos los recursos disponibles para generar un ambiente educativo más valioso para el estudiante. Los programas educativos de instituciones de educación superior exhiben diferentes capacidades. Si estas capacidades complementarias son compartidas entre instituciones por medio de ejercicios de simulación los horizontes del estudiante pueden expandirse. Finalmente, un modelo de simulación situacional de uso general puede servir como un ambiente valioso para el desarrollo de una variedad de proyectos de investigación. Debido a su flexibilidad y amplio alcance, un modelo de simulación situacional de uso general se puede utilizar como un laboratorio virtual para realizar experimentos y para evaluar hipótesis.

\section{Un sistema de simulación de uso general para la gerencia de la construcción}

Para explicar el funcionamiento interno de un ambiente de simulación situacional de uso general para la gerencia de la construcción es necesario explorar lo que significa tal ambiente desde el punto de vista de participantes y de desarrolladores. Para el propósito de este artículo, el término participantes se refiere a los individuos que experimentan el ambiente simulado (sin importar su nivel de conocimientos), mientras que el término desarrolladores se refiere a los individuos que diseñan y construyen los ambientes.

\subsection{El Punto de vista del participante}

Los participantes en un ambiente situacional de uso general que simula el proceso de gerencia de la construcción realizan tres actividades básicas: la comprensión del proyecto, el monitoreo del proyecto, y la gerencia del proyecto (Figura 1). La comprensión del proyecto es una tarea sin limitación de tiempo donde los participantes se familiarizan con el proyecto que administrarán. Ellos logran este objetivo revisando la información desde perspectivas diversas tales como motivación, diseño, programa, y presupuesto. Algunos ejemplos de la clase de información específica que estas perspectivas ofrecen se ilustran en la Figura 1. La motivación se relaciona con las razones del dueño a impulsar el proyecto. La perspectiva del diseño ofrece los detalles específicos del proyecto. La perspectiva del programa presenta los aspectos de programación de la obra. Finalmente, la perspectiva del presupuesto familiariza a los participantes con los costos del proyecto. 0 tras perspectivas pueden también formar parte de esta etapa, pero estas cuatro son consideradas como las áreas fundamentales.

El monitoreo del proyecto comienza tan pronto como el participante inicia la fase de la construcción de la obra. A partir de este punto, el proyecto avanza en intervalos de tiempo de la misma magnitud y la información sobre el proyecto se proporciona al final de 
cada intervalo. Es importante que el tiempo avance en intervalos constantes para presentar un panorama más realista al participante y permitir cálculos matemáticos y verificación de restricciones de forma constante. Los datos pertinentes se comparan con los valores encontrados en los planes originales tal como se muestra en la Figura 1. Algunos ejemplos de los parámetros de evaluación tradicional que se presentan al participante para su análisis incluyen el índice de efectividad en la programación, el índice de efectividad de costos, y el índice de la productividad entre otros.

La gerencia del proyecto es concomitante con el monitoreo del mismo. En ausencia de una situación (acontecimiento), los participantes tienen dos opciones: acción o inacción. La inacción del participante permite que la simulación continúe ininterrumpidamente. Sin embargo, si el participante decide que hay una oportunidad de mejorar la eficiencia del proceso, la simulación se detiene brevemente para proporcionar tiempo al participante para realizar sus acciones. Cuando se presenta una situación, el participante recibe la información relevante a la naturaleza y la severidad de la situación o evento y la simulación se detiene brevemente por un período específico para permitir la reacción a tal situación y reducir al mínimo sus consecuencias adversas.

Finalmente, la sección del nuevo ciclo en la

Figura 1 representa la naturaleza iterativa de la simulación mientras que el reloj avanza de un intervalo al siguiente. El monitoreo y la gerencia del proyecto continúa a través de todos los ciclos en la simulación. La simulación termina cuando todas las actividades en el proyecto concluyen.

\subsection{El Punto de vista del desarrollador}

Los desarrolladores de simulaciones en un ambiente situacional que simula el proceso de gerencia de la construcción realizan cuatro actividades básicas: la definición del proyecto, el diseño de situaciones, la selección de situaciones, y la definición del ciclo de retroalimentación (Figura 2). La definición del proyecto implica la selección de la obra que se simulará así como la creación de los documentos que los participantes requerirán durante la fase de comprensión del proyecto.

El diseño de situaciones es la base de un ambiente situacional de uso general. La flexibilidad en el diseño de situaciones es lo que permite que el ambiente sea de uso general en lugar de ser de propósito específico. Los diseñadores deben de tomar decisiones con respecto a los escenarios a simular, las acciones, y las relaciones entre variables. El escenario es la descripción de la situación en su contexto apropiado, lo que requiere de una narrativa y de una cuenta regresiva y puede también beneficiarse de información audiovisual (fotografías, diagramas, videos, sonidos, animaciones, etc.). La narrativa y la información audiovisual explican la naturaleza y la severidad del problema, mientras que la cuenta regresiva determina el tiempo que un participante tiene para analizar el problema y reaccionar al mismo. Las acciones determinan las consecuencias de la situación en las variables, las restricciones, y las dependencias del modelo. Las acciones traducen la naturaleza imprecisa de una situación en efectos específicos sobre el proyecto. Por ejemplo, una situación tal como una tormenta de invierno con cantidades significativas de nieve puede tener como acciones una reducción del $50 \%$ en la productividad de las actividades al aire libre y un retraso de tres días en la entrega de materiales. Por lo tanto, los diseñadores 0 desarrolladores deben de establecer la relación de causaefecto entre los acontecimientos y las acciones. El modelo no dicta estas relaciones para ofrecer la mayor flexibilidad posible. Los desarrolladores pueden basar estas relaciones en teorías, datos empíricos, inventivas heurísticas, o en consejos de experto entre otros métodos.

La selección de situaciones es el proceso de elección de las situaciones que afectarán una simulación particular. Estas situaciones pueden ser creadas en la fase de diseño o importadas de la biblioteca de situaciones creada por la comunidad de desarrolladores. Durante la fase de selección de situaciones, los desarrolladores también especifican las relaciones entre situaciones con respecto a su yuxtaposición potencial para mantener integridad lógica. Por ejemplo, la situación descrita previamente con respecto a una tormenta de invierno no puede suceder al mismo tiempo que otra situación relacionada con movimientos de tierras debido a que el movimiento de tierras en medio de una tormenta de invierno severa no es factible dadas las condiciones atmosféricas.

Los desarrolladores también deben de especificar el ciclo de retroalimentación. El ciclo de retroalimentación se puede definir como la frecuencia con que la información es presentada a los participantes. Una simulación puede ofrecer datos en tiempo real o con algún retraso o una combinación de ambos casos. La definición de ciclo de retroalimentación también incluye la decisión con respecto a qué tipo de información estará disponible para los participantes. 


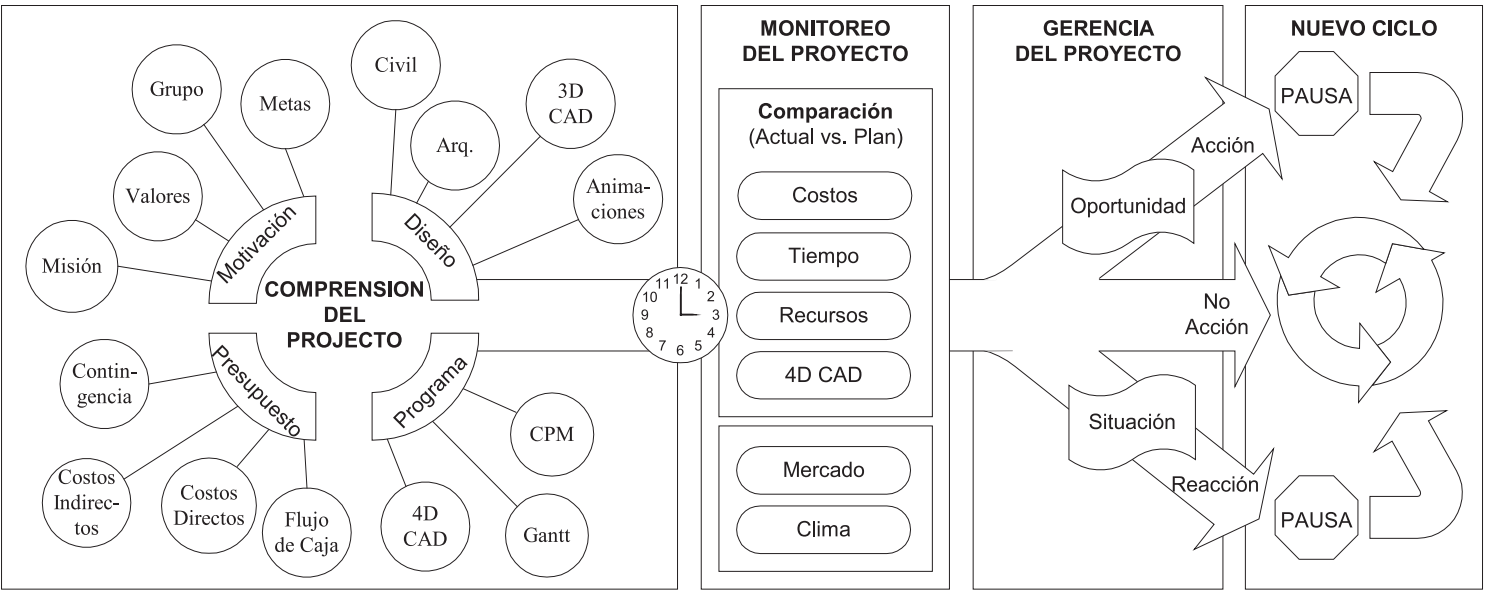

Figura1. Punto de visa del participante

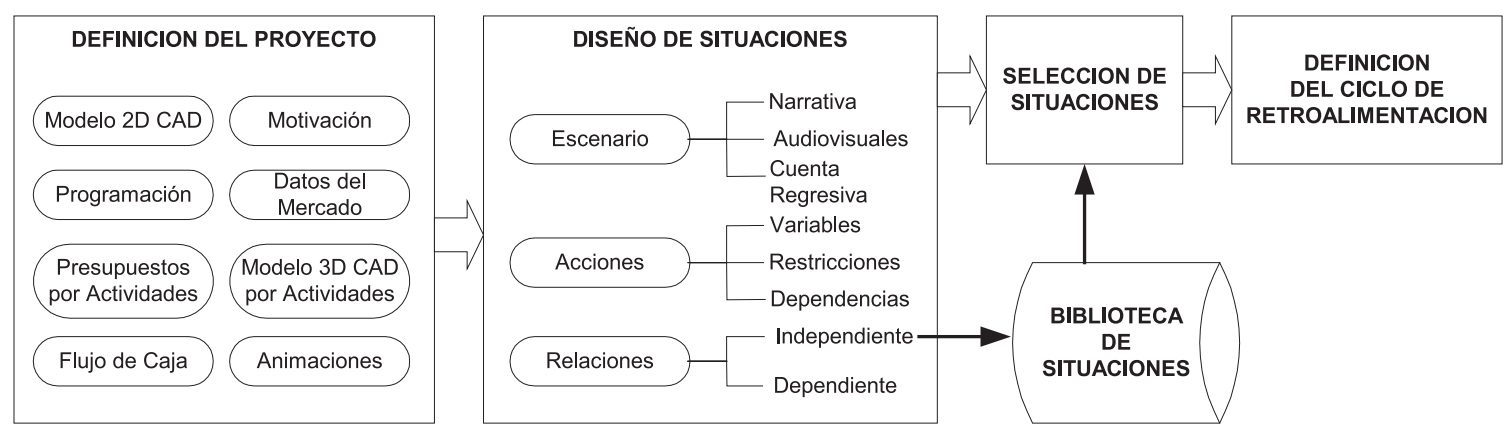

Figura 2. Punto de vista del desarrollador

\section{Implementación del "virtual coach"}

Anteriormente discutimos que la programabilidad es una de las ventajas de un ambiente de simulación situacional de uso general. Sin embargo, al examinar el punto de vista del desarrollador no mencionamos nada relacionado con la habilidad de los desarrolladores para cambiar las relaciones en el ambiente tal como el formalismo que determina la duración remanente de actividades en ejecución. Esta omisión fue intencional, pues queremos mantener la conceptualización y la implementación del ambiente como dos actividades independientes. Cada vez que utilizamos la palabra "simulación," una imagen de un sistema de cómputo se desarrolla en nuestras mentes. Sin embargo, el concepto "simulación" no es un sinónimo de computación dado que ambientes de simulación pueden ser creados sin utilizar computadoras. Las actividades, los modelos, y las relaciones representadas en las Figuras 1 y 2 pueden ser realizados y ejecutados sin utilizar un sistema de cómputo. De forma tal que estos diagramas sirven como la base conceptual en la que se pueden desarrollar diferentes sistemas de cómputo para automatizar el ambiente. El "Virtual Coach" es un ejemplo de un sistema de cómputo desarrollado por los autores. Este representa 
la migración del modelo conceptual a la implementación de un sistema de simulaciones situacionales de uso general.

La versión actual del sistema es muy limitada en su implementación y sirve el propósito de ser un sistema piloto. El ambiente de simulación toma en cuenta pocas variables así como un número limitado de acontecimientos. Además, la interactividad de los participantes con el programa es limitada a la asignación de recursos existentes y a la compra/contrato de nuevos recursos. Versiones futuras incluirán más variables y representarán el ambiente en más detalle. Colaboración futura con la industria de la construcción aumentará el número de escenarios y acontecimientos prácticos dentro de la estructura existente. La implementación actual simplemente valida la conceptualización y proporciona una plataforma para realizar estudios preliminares y determinar cómo es que los estudiantes reaccionan al sistema. Versiones futuras desarrollarán también una interfase de cómputo considerablemente más sofisticada. La interfase actual sólo proporciona los elementos básicos requeridos para ejecutar la simulación. Visualización futura presentará información contextual audiovisual, gráficos del sitio de la obra en 4D (3 dimensiones espaciales más tiempo) y permitirá también al participante planificar dinámicamente el proyecto simulado.

\section{Evaluación del "virtual coach"}

El objetivo de nuestro trabajo experimental es determinar si un sistema de simulaciones situacionales tal como el "Virtual Coach" se puede utilizar para educar futuros gerentes de construcción. Además, quisimos aprender sobre el proceso de aprendizaje en el área de la gerencia de la construcción basándonos en nuestras observaciones de cómo, si del todo, estos ambientes pueden utilizarse para entender el proceso cognitivo en la gerencia de la construcción.

Una implementación piloto del "Virtual Coach" fue evaluada con un grupo de 19 estudiantes de gerencia de la construcción, como parte de una clase en Administración de Proyectos en la Universidad de Washington. Los estudiantes tomaron exámenes antes y después de experimentar la simulación y se les solicitó "pensar en voz alta" sobre sus decisiones y percepciones de lo que sucedía durante el ejercicio. Todos los comentarios hechos durante la simulación fueron grabados y analizados. Los exámenes pre y post-experimentación requirieron que los estudiantes situaran (en una escala de 1-10), en su opinión, la importancia de una lista de factores en el desarrollo de un plan para la construcción de un proyecto con una duración de 12 semanas. Los estudiantes también revisaron una lista de limitaciones que gobiernan el escenario así como toda la información necesaria para administrar el proyecto. Las limitaciones incluyeron consideraciones del programa de actividades (CPM ), limitaciones del presupuesto y la posibilidad de acontecimientos tales como mal tiempo, demoras en la entrega de materiales y la escasez de mano de obra calificada.

Cuatro de las calificaciones asignadas por los estudiantes, antes de y después de utilizar la simulación, fueron sumadas y comparadas utilizando la prueba estadística t-student. Las calificaciones escogidas para el análisis fueron aquellas que relacionaron las limitaciones de programación y de recursos con la necesidad de anticipar la demora en un proyecto (dando prioridad a actividades críticas en caso de una demora, brindando atención a restricciones de espacio en el sitio de la obra, anticipando las demoras futuras en la entrega de materiales, acelerando las actividades para crear el búfer para una demora anticipada, etc.). La diferencia entre las calificaciones fue estadísticamente significativa, como se muestra en la Tabla 1.

Tabla 1. Resultados de los estudiantes en pre- y postexperimentación

\begin{tabular}{||c|c|c||}
\hline & Promedio & Desviación Estándar \\
\hline Pre- Experimentación & 21.26 & 4.92 \\
\hline Post- Experimentación & 25.31 & 4.70 \\
\hline \multicolumn{2}{|c|}{ T-estadística: $\mathrm{t}(18)=3.32, \mathrm{p}<.01$} \\
\hline
\end{tabular}

Es interesante resaltar que las preguntas en las que los estudiantes mostraron una mejora significativa trataron los temas de la satisfacción de limitaciones de recursos y limitaciones temporales, lo que sugiere que los estudiantes aprenden al comprender las limitaciones fundamentales que se presentan en un proyecto y al entender las consecuencias de sus violaciones.

Un análisis cualitativo de los "pensamientos" de los participante (tal como se grabaron durante la simulación) y la información brindada en un cuestionario nos proporcionó con datos valiosos con respecto a lo que los estudiantes aprendieron de la experiencia. A continuación se presenta una lista de reacciones de estudiantes después de que experimentaron la simulación. 
Estas han sido transcritas "al pie de la letra" y son representativas de la reacción general que obtuvimos de los estudiantes. Las respuestas acentúan el hecho que lo que impresionó a la mayoría de los estudiantes fue la habilidad de obtener la "visión general y comprensiva del proyecto" y las relaciones entre la mano de obra, el presupuesto, la programación de actividades y el impacto de sus decisiones en el ambiente.

Participante 1: Me gustó el hecho de que pudiera ver lo que mis acciones hacían al presupuesto y a la programación de la obra. En la industria uno siempre trata de rescatar unos pocos días críticos al inicio para contrarrestar los problemas imprevistos en el futuro.

Participante 2: El "Virtual Coach" fue una buena simulación y puso juntos los elementos críticos de administración de un proyecto, del trabajo, de los materiales, de la programación, y del costo. Yo siento que proporcionó una buena manera de realmente controlar la programación y ver el efecto que un cambio puede tener en todas las variables.

Participante 3: Me gustó como el proyecto no siguió el plan original. Pienso que fue una manera buena de comunicar cómo acontecimientos cómo eventos impredecibles suceden por lo que uno necesita cambiar la manera en la que se tratan las limitaciones ya existentes.

Participante 4: El "Virtual Coach" hace un buen trabajo al dar a los estudiantes una mejor idea de la visión general. ... Sentía como que necesitaba entender cómo es que las relaciones entre la asignación de materiales y de mano de obra fueron determinadas y utilizadas, antes de que yo pusiera realmente mucha confianza en el "Virtual Coach."

Participante 5: Pensé que el "Virtual Coach" es realmente interesante en el hecho de que tomó en cuenta los muchos parámetros externos que pueden afectar el proceso de la construcción de un proyecto.

Participante 6: La asignación de los costos y la programación de la obra en tiempo real permiten que uno vea dónde es que estas variables están y para dónde es que van.

Participante 7: Pienso que el "Virtual Coach" es una gran actividad para nosotros. Lo pone a uno a pensar acerca de los recursos, la administración, la estimación de costos y la programación del proyecto de forma conjunta. Pienso que podría ser mejorado permitiéndonos ver las diferencias en los costos por unidad, y cuánto es que nuestras decisiones afectan el proyecto. Por ejemplo, me gustaría ver cuánto más costoso es el emplear a un trabajador pagando un extra. De otra forma es un gran programa, ningún trabajo escolar que he hecho me ha enseñado a manejar recursos tan bien como este programa.

Las reacciones grabadas de los participantes durante la simulación nos ayudaron a identificar los varios momentos de sorpresa en los estudiantes que refuerzan nuestra opinión de que la simulación ayuda a los estudiantes a lograr una visión sistémica del proceso constructivo. Es interesante recalcar cómo es que los estudiantes reaccionaron a demoras en la ejecución de la obra. La reacción más común fue el acelerar la actividad del momento, sin verificar dónde es que esta estaba en la ruta crítica. Había también una tendencia general a aumentar la cantidad de la mano de obra en una actividad que estaba demorada sin anticipar la reacción potencial en términos de baja calidad del producto o la ausencia de disponibilidad de la misma mano de obra para trabajar fuerte en el futuro. Fue también interesante el notar un comentario de un estudiante cuando se dio cuenta de que un proyecto afectado por un acontecimiento hacia el final del mismo es mucho más difícil de recuperar que un proyecto que se demoró al inicio.

Los momentos de sorpresa sucedieron cuando una reacción inesperada forzó a los participantes a analizar de nuevo una decisión tomada anteriormente en la simulación. La comprensión de que existe una demora entre una acción y su reacción motivó a los estudiantes a tomar una pausa y determinar que tan sensible era el sistema a sus decisiones. Además, esta realización los ayudó a percibir que los problemas a menudo no son simplemente un disturbio localizado, pero los resultados de relaciones causales estructurales, que son recíprocas por su misma naturaleza.

\section{Conclusiones}

Basado en los resultados cual itativos obtenidos de los cuestionarios de los estudiantes, donde 16 de los 19 participantes opinaron que el "Virtual Coach" es un instrumento educativo valioso; las diferencias estadísticamente significativas de los exámenes de prey post-experimentación; y el alto valor de confianza ilustrado en la Tabla 1; se puede concluir que una 
intervención que utiliza simulaciones situacionales puede ser útil en la educación de los futuros gerentes de construcción.

De nuestro estudio también concluimos que los estudiantes tienden a aprender mejor cuando comprenden las limitaciones fundamentales presentes en un proyecto de construcción y las consecuencias que sus decisiones pueden tener cuando las limitaciones son violadas. N uestro análisis determinó que los estudiantes opinan que la mejor forma de lidiar con tales infracciones es desarrollando una vista sistémica de la gerencia de la construcción y una mejor comprensión de las relaciones causales entre variables.

Basados en la reacción entusiasmada que recibimos de los estudiantes y nuestro análisis de sus interacciones con el sistema, nos parece conveniente continuar el desarrollo de sistemas de aprendizaje dinámicos tales como el "Virtual Coach," ya que pueden ser instrumentos útiles en el proceso educativo de los futuros gerentes de la construcción.

Finalmente, podemos concluir que las simulaciones situacionales de uso general son instrumentos que los educadores de la gerencia de la construcción pueden utilizar para transmitir, en una manera dinámica, las relaciones complejas entre las variables presentes en la mayoría de los proyectos de construcción. Estas simulaciones pueden ser una adición bienvenida al grupo de herramientas del educador de la ingeniería y servir su propósito al lado otros enfoques pedagógicos.

\section{Referencias}

AbouRizk S. (1993), "Stochastic simulations of construction bidding and project management," Microcomputers in Civil Engineering, 8, 343-353.

AbouRizk S. y Sawhney A. (1994), "Simulation and gaming in construction engineering education," $A S E E / C^{2} E^{2} / C^{2} E I$ Conference, Edmonton, Alberta.

Barab S.A., H ay K.E., Barnett M. y Squire K. (2001), "Constructing virtual worlds: tracing the historical development of learner practices," Cognition and Instruction, 19 (1), 47-94.

Bertz M. y Baker N.C. (1996), "CELL - a vertically integrated learning resource," Proceedings of the Third Congress on Computing in Civil Engineering, ASCE, Anaheim, 348-354.

Brown J.S., Collins A. y Duguid P. (1989), "Situated cognition and the culture of learning," Educational Researcher, 18 (1), 32-42.
Chi M., Bassok M., Lewis M., Reimann P. y Glaser R. (1989), "Self-explanations: how students study and use examples in learning to solve problems," Cognitive Science, 13, 145-182.

Chinowsly y Vanegas (1996), "Combining practice and theory in construction education curricula," 1996 ASEE Annual Conference Proceedings.

Echeverry D. (1996), "Mulimedia-based instruction of building construction," Proceedings of the Third Congress on Computing in Civil Engineering, ASCE, Anaheim, 972-977.

Fruchter R. (1997), "The A/E/C virtual atelier: experience and future directions," Proceedings of the Second Congress of Computing in Civil Engineering, ASCE, Atlanta, 441-448.

Halpin D. y Woodhead R. (1970), "A Computerized Construction Management Game." Department of Civil Engineering, University of Illinois, U rbana Champagne.

Jaafari A., Manivong K. y Chaaya M. (2001), "VIRCO N : interactive system for teaching construction management," Journal of Construction Engineering and Management, 127 (1), 66-75.

McCabe B., Ching K.S. y Savio R. (2000), "STRATEGY: a construction simulation environment," Proceedings of Construction Congress VI, 115-120.

N dekugri I. y Lansley P. (1992), "Role of simulation in construction management." Building Research and Information. 20 (2), 109-115.

NSF (1995), "Restructuring engineering education: a focus on change," Report of the 1994 NSF Workshop on Engineering Education, Division of U ndergraduate Education, Directorate for Education and Human Resources, N ational Science Foundation, Washington D.C.

Pennell R., Durham M., Ozog C. y Spark A. (1997), "W riting in context: situated learning on the web," Proceedings of the $14^{\text {th }}$ Annual ASCILITE Conference, Perth,WA, 463-469.

Sawhney A., M und A. y Koczenasz J. (2001), “Internetbased interactive construction management learning system," Journal of Construction Education, 6 (3), 124138. 